REVISTA DE DERECHO UNED, NÚM. 26, 2020

\title{
PROPUESTAS DE REFORMA PARA FOMENTAR LA NATALIDAD, LA CONCILIACION Y LA FAMILIA EN EL IRPF
}

\author{
PROPOSALS TO REFORM THE PERSONAL INCOME TAX AND \\ PROMOTE AND PROTECT THE NATALITY, WORK-LIFE \\ CONCILIATION AND FAMILY
}

\section{LuZ Martinez OTERO ${ }^{1}$}

Resumen: España está atravesando una época de grave crisis demográfica y, paradójicamente, en la actualidad, existe una brecha entre maternidad/paternidad deseada y maternidad/paternidad real. Existen diferentes barreras socioeconómicas que contribuyen a que los españoles tengan que sacrificar su deseo de tener más hijos, una de las más importantes, según ha quedado recientemente acreditado por la encuesta publicada por el Instituto Nacional de Estadística (INE. Encuesta de Fecundidad de 28 de Noviembre de 2018), es la dificultad que encuentran los españoles a la hora de conciliar su trabajo profesional con su vida personal.

Los tributos tienen una finalidad extra tributaria y pueden ser un instrumento de política fiscal que proteja y promueva los valores protegidos en la Constitución, por ejemplo la familia. Mediante políticas fiscales adecuadas se pueden proteger valores como la maternidad, la paternidad, la conciliación, la corresponsabilidad, la igualdad, la infancia y en definitiva, la familia.

El presente artículo analiza el régimen tributario de las prestaciones por maternidad recientemente modificado tras la sentencia del Tribunal Supremo número 1482/2018, profundiza en el papel de

1 Abogada. Asesor Fiscal. Alumna del Programa de Doctorado en Derecho y Ciencias Sociales de la Universidad Nacional de Educación a Distancia (UNED)

(C) UNED. Revista de Derecho UNED, núm. 26, 2020 
la familia como agente de cambio capaz de revertir el problema demográfico que atraviesa nuestro país y propone medidas tributarias para reformar el Impuesto sobre la Renta de las Personas Físicas (IRPF), medidas que promueven la conciliación, fomentan la natalidad, protegen la paternidad y la maternidad y facilitan el cuidado de los hijos y la atención a las familias.

Palabras clave: Impuesto sobre la Renta de las Personas Física, Familia, Natalidad, Conciliación, Maternidad, Paternidad, Corresponsabilidad, Igualdad.

Abstract: Spain suffers from a major demographical crisis, but, paradoxically, there is a gap between desired and real parenthood as well. There are many socio-economical barriers which contribute to Spaniards giving up their willingness to have children, one of the most important of which is the difficulty to keep a work-life balance (INE 2018).

Taxes can become an instrument to a fiscal policy that promotes and protects constitutional values, for instance, the family. Through appropriate fiscal policies taxes can promotes and protects parenthood, work-life conciliation, co-responsibility, equality, childhood and, ultimately, family.

This article analyzes the tax regime of maternity benefits recently modified after the Supreme Court 1482/2018, deepens the role of the family as an agent of change capable of reversing the demographic problem that our country is going through and proposes measures tax to reform the Personal Income Tax, measures that, by means of facilitating work-life conciliation, promote natality and remove difficulties from children and family care.

Keywords: Personal Income Tax, Family, Natality, Work-life balance, Maternity, Paternity, Co-responsibility, Equality.

Recepción original: 6-2-2020

Aceptación original: 11-6-2020

Sumario: I. Planteamiento. II. Régimen tributario por maternidad y paternidad en el IRPF. III. Situación demográfica en nuestro país. El papel de la familia como agente de cambio. IV. Propuestas para proteger la familia, la maternidad y la conciliación. IV.A. Planteamiento. IV.B. Mínimo personal y familiar. IV.C. Prestación universal por nacimiento. IV.D. Exención de la prestación por maternidad. IV.E. Deducciones familiares (estatales y universales). IV.E.1. Deducción por reducción de jornada para conciliar vida personal y vida profesional. IV.E.2. Deducción por gastos de guardería. IV.E.3. 
Deducción por gastos de ayuda doméstica. IV.E.4. Deducción por gastos de escolaridad. IV.E.5. Deducción por transporte escolar. IV.F Deducciones de la Cuota Diferencial. IV.F.1. Deducción por maternidad. IV.F.2. Deducción para familias numerosas o personas con discapacidad a cargo. V. Necesidad de análisis cuantitativos sobre los efectos económicos de las medidas propuestas. VI. Conclusiones

\section{PLANTEAMIENTO}

En el presente artículo se pretende formular propuestas de reforma en el Impuesto sobre la Renta de las Personas Físicas para promover y fomentar la natalidad, la conciliación y para proteger la institución familiar y así implementar políticas fiscales que pongan en valor la familia.

Se formulan estas propuestas en un momento en el que España atraviesa una crisis demográfica sin precedentes que paradójicamente convive con el deseo de muchas familias españolas de tener más hijos, pero éstas se encuentran con obstáculos sociales, económicos y laborales, entre otros, y con poco apoyo institucional y escasas ayudas públicas a la natalidad y a la conciliación ${ }^{2}$.

En primer lugar se analiza el régimen tributario de las prestaciones por maternidad y paternidad que recientemente ha sido objeto de un cambio importante tras la Sentencia del Tribunal Supremo número 1462/2018, de 3 de octubre de 2018.

En segundo lugar, se hace una breve reflexión sobre el papel de la familia como agente de cambio en la delicada situación demográfica que atraviesa nuestro país ${ }^{3}$, analizando precisamente esa brecha

2 "Las razones laborales o de conciliación de la vida familiar y laboral y las económicas son las más importantes por las que las mujeres han tenido menos hijos de los deseados" Instituto Nacional de Estadística, Encuesta de Fecundidad, 28 de noviembre de 2018, pág. 2 .

3 "Durante las últimas décadas, se han producido cambios importantes en los comportamientos sociales, que han dado lugar a modificaciones en las principales variables demográficas en España. Los datos del Instituto Nacional de Estadística (INE) nos vienen alertando, especialmente, del envejecimiento de la población, derivado fundamentalmente del aumento de la esperanza de vida y de la falta de nacimientos. Esta realidad no se produce exclusivamente en España: estamos ante un fenómeno generalizado en Europa, aunque en algunos países de nuestro entorno, y desde hace alguna década, ya se están tomando medidas para tratar de evitar las consecuencias sociales, económicas e incluso políticas que tienen estos cambios". 
entre maternidad deseada y maternidad real ${ }^{4}$ y enumerando algunas de las principales barreras que encuentran las familias españolas para tener más hijos.

En tercer lugar, se exponen nuestras propuestas para reformar el Impuesto sobre la Renta de las Personas Físicas y para conseguir proteger a través del sistema tributario a la institución familiar, la maternidad, la paternidad, la infancia, la conciliación y la corresponsabilidad en el seno de todas las familias y así, darles un mayor valor, reconociendo su papel fundamental en la sociedad y en el futuro de nuestra nación.

Finalmente se propone la necesidad de cuantificar el efecto de nuestras propuestas para poder implementar las que verdaderamente cumplan con su objetivo (la protección a la institución familiar) y se exponen las conclusiones.

\section{RÉGIMEN TRIBUTARIO POR MATERNIDAD Y PATERNIDAD EN EL IRPF}

El Real Decreto-Ley 27/2018, de 28 de diciembre, por el que se adoptan determinadas medidas en materia tributaria y catastral, publicado en el Boletín Oficial del Estado el 28 de Diciembre de 2018, modificó el artículo 7 h) de la Ley 35/2006, de 28 de noviembre, del Impuesto sobre la Renta de las Personas Físicas (en adelante, LIRPF), en lo relativo a las prestaciones públicas por maternidad percibidas de la Seguridad Social, que la Sentencia del Tribunal Supremo número 1462/2018 de 3 de Octubre, declaró exentas.

En la Exposición de Motivos del Real Decreto-Ley se justifica la utilización de este instrumento jurídico del real decreto-ley diciendo que "Debe tenerse en cuenta que la extensión del cambio normativo a ejercicios anteriores no prescritos justifica, en el presente caso, la extraordinaria y urgente necesidad que constituye el presupuesto habilitante para acudir al instrumento jurídico del real decreto-ley, permi-

LÓPEZ LÓPEZ, M.T. y GONZÁLEZ HINCAPIÉ, V. “Tener hijos, ¿forma parte del proyecto vital de los madrileños?. Maternidad, paternidad y trabajo remunerado", Comunidad de Madrid, 2017, pág. 5.

4 "Las razones de esta brecha están normalmente ligadas a la falta de equilibrio entre el trabajo y la vida personal y familiar; al retraso de la edad en la que se produce la maternidad; o al elevado coste de la vida". CHINCHILLA, N. Maternidad y trayectoria profesional. Análisis de las barreras e impulsores para la maternidad de las mujeres españolas. Estudio 2017, ICWF - Centro Internacional Trabajo y Familia, 2017, pág. 41. 
tiendo que se pueda solicitar la devolución del impuesto soportado antes de que prescriba el ejercicio en el que se declararon las retribuciones que ahora se declaran exentas".

El origen de esta cuestión controvertida se remonta al año 2007. Desde entonces se venía discutiendo ante los tribunales si la prestación por maternidad recibida de la Seguridad Social está o no exenta del Impuesto sobre la Renta de las Personas Físicas, esto es, si esa prestación por maternidad estaba contemplada en el artículo 7 h) de la LIRPF. Distintos órganos administrativos y tribunales se pronunciaron en sentido contradictorio. Así, el Tribunal Económico Administrativo Regional de Madrid, en sus Resoluciones de 23 de abril de 2007 y 18 de febrero de 2014 desestimó la exención de la prestación por maternidad, mientras que el Tribunal Superior de Justicia de Madrid, estimó la exención de la prestación por maternidad en su Sentencia del 6 de julio de 2016. El Tribunal Económico Administrativo Regional de Andalucía (Sevilla) en su Resolución 20 de febrero de 2015 desestimó la exención de la prestación por maternidad del mismo modo que lo hizo el Tribunal Superior de Justicia de Andalucía en su Sentencia de 27 de octubre de 2016.

Ante los distintos pronunciamientos y dada la inseguridad jurídica y la polémica existente, el Tribunal Supremo se pronunció en casación mediante la Sentencia del 3 de octubre de 2018 y fijó como doctrina legal que "las prestaciones públicas por maternidad percibidas de las Seguridad Social están exentas del Impuesto sobre la Renta de las Personas Físicas".

Como consecuencia de la Sentencia 1462/2018, surgieron inmediatamente algunos interrogantes, tales como la posibilidad de que estuvieran asimismo exentas la prestación por paternidad y aquellas prestaciones que funcionarios públicos o profesionales dados de alta en mutualidades alternativas a la Seguridad Social percibían como consecuencia de su maternidad (o paternidad). Así, el legislador tributario quiso actualizar el artículo $7 \mathrm{~h}$ ) LIRPF y esclarecer esos otros supuestos de hecho que por ser casi idénticos y para evitar discriminaciones y una vulneración del principio de igualdad también deberían quedar exentos, por lo que se promulgó el Real DecretoLey 27/2018 modificando la redacción del artículo 7 h) LIRPF que quedó redactado como sigue:

«h) Las prestaciones por maternidad o paternidad y las familiares no contributivas reguladas, respectivamente, en los Capítulos VI y VII del Título II y en el Capítulo I del título VI del texto refundido de la Ley General de la Seguridad Social, aprobado por el Real Decreto Legislativo 8/2015, de 30 de octubre y las pensiones y los haberes pa- 
sivos de orfandad y a favor de nietos y hermanos, menores de veintidós años o incapacitados para todo trabajo, percibidos de los regímenes públicos de la Seguridad Social y clases pasivas.

Asimismo, las prestaciones reconocidas a los profesionales no integrados en el régimen especial de la Seguridad Social de los trabajadores por cuenta propia o autónomos por las mutualidades de previsión social que actúen como alternativas al régimen especial de la Seguridad Social mencionado, siempre que se trate de prestaciones en situaciones idénticas a las previstas en el párrafo anterior por la Seguridad Social para los profesionales integrados en dicho régimen especial. La cuantía exenta tendrá como límite el importe de la prestación máxima que reconozca la Seguridad Social por el concepto que corresponda. El exceso tributará como rendimiento del trabajo, entendiéndose producido, en caso de concurrencia de prestaciones de la Seguridad Social y de las mutualidades antes citadas, en las prestaciones de estas últimas.

En el caso de los empleados públicos encuadrados en un régimen de Seguridad Social que no de derecho a percibir la prestación por maternidad o paternidad a que se refiere el primer párrafo de esta letra, estará exenta la retribución percibida durante los permisos por parto, adopción o guarda y paternidad a que se refieren las letras a), b) y c) del artículo 49 del texto refundido de la Ley del Estatuto Básico del Empleado Público, aprobado por el Real Decreto Legislativo $5 / 2015$, de 30 de octubre o la reconocida por la legislación específica que le resulte de aplicación por situaciones idénticas a las previstas anteriormente. La cuantía exenta de las retribuciones o prestaciones referidas en este párrafo tendrá como límite el importe de la prestación máxima que reconozca la Seguridad Social por el concepto que corresponda. El exceso tributará como rendimiento del trabajo.

Igualmente estarán exentas las demás prestaciones públicas por nacimiento, parto o adopción múltiple, adopción, maternidad o paternidad, hijos a cargo y orfandad.»

Con el Real Decreto-Ley se pusieron fin a los mencionados interrogantes según explicaba en su exposición de motivos:

"Existen otros colectivos que perciben igualmente prestaciones por paternidad o maternidad que al no estar amparados ni por dicha sentencia ni por la regulación actual del Impuesto tendrían que tributar por este último, lo que provocaría una situación de clara discriminación difícilmente justificable.

En concreto, tales colectivos son, por una parte, los empleados públicos (...) y los profesionales no integrados en el régimen especial de la Seguridad Social de los trabajadores por cuenta propia o autónomos que perciben tales prestaciones de las mutualidades (...)

En consecuencia, la nueva redacción que se introduce en la Ley del Impuesto, además de declarar expresamente exentas las prestaciones 
por maternidad o paternidad satisfechas por la Seguridad Social, extiende la exención a las prestaciones percibidas por los otros colectivos señalados, hasta el límite de la prestación máxima que la Seguridad Social reconoce por tal concepto.

Posteriormente, en enero de 2019, la Dirección General de Tributos ha emitido algunas consultas vinculantes en las que, a la luz de la nueva redacción del artículo 7 h) LIRPF, explica cómo las prestaciones públicas por paternidad percibidas de la Seguridad Social están exentas ${ }^{5}$, o como las prestaciones percibidas por los funcionarios durante su permiso de paternidad o maternidad también están exen$\operatorname{tas}^{6}$. Son cuestiones que aún quedando perfectamente claras en el nuevo artículo 7 h) LIRPF, la Dirección General de Tributos ha querido precisar.

Dejando a un lado el análisis de la Sentencia y nuestra opinión particular sobre la misma, así como la nueva redacción del artículo 7 h) LIRPF, y tomando como punto de partida la exención de la prestación por maternidad y paternidad, nos gustaría aprovechar este momento, en el que el propio Tribunal Supremo se ha pronunciado a favor de la maternidad recordando en la propia Sentencia 142/2018 que "la maternidad es una situación protegida (así como la adopción, el acogimiento familiar y la familia en sentido amplio)", para estudiar la posibilidad de implementar medidas fiscales en pro de la maternidad y la familia.

\section{SITUACIÓN DEMOGRÁFICA EN NUESTRO PAÍS. EL PAPEL DE LA FAMILIA COMO AGENTE DE CAMBIO}

En las últimas décadas se han producido en España dos fenómenos paralelos que han propiciado cambios sociales y demográficos con sus consiguientes consecuencias económicas y sociales. Nos referimos al aumento de la esperanza de vida y a la caída de la natalidad. Nos encontramos con una población envejecida y con un verdadero problema en cuanto al reemplazo generacional; nuestra pirámide poblacional es una pirámide invertida. España lleva varias décadas por debajo de la fertilidad mínima que se requiere para asegurar el reemplazo generacional. ${ }^{7}$

5 DGT 0130/2019

DGT 0134/2019

"La caída de la fecundidad sitúa a España -y dentro de ella a la CM- en el umbral de lo que los que los demógrafos han denominado "fecundidad muy baja", refiriéndose con ello a cifras por debajo de 1,3 hijos por mujer". BILLARI, F. y KOHLER, H.P., "Patterns of low and lowest-low fertility in Europe," Population 
Aunque el hecho de que las familias decidan o no tener hijos es un asunto estrictamente privado, no podemos olvidar que tiene consecuencias sociales y por tanto los poderes públicos y la sociedad en general no pueden, ni deben, ser indiferentes ante esta realidad. La composición de la población y el capital humano juegan un papel clave en el crecimiento económico; tienen implicaciones sobre las políticas públicas y afectan al actual estado de bienestar, muy especialmente al sistema de pensiones, ya que al tratarse de un modelo de reparto, exige un crecimiento de la población para su sostenibilidad; y la caída en el número de nacimientos también afecta al bienestar de la sociedad y de las familias. Existe consenso a la hora de aceptar que hay razones económicas y sociales que justifican la necesidad de intervenciones públicas en relación con la natalidad, pero sobre lo que ya no existe tanto acuerdo es sobre las medidas o actuaciones que deberían ponerse en marcha ${ }^{8}$.

El problema de la baja natalidad resulta agravado cuando estudiamos las estadísticas que demuestran un importante desfase entre la fertilidad real y la fertilidad deseada. Así, eliminar las barreras que impiden tener el número de hijos que se desea, no solo es facilitar la culminación de un proyecto vital y personal, sino garantizar la riqueza y la sostenibilidad del país en el medio y largo plazo9.

Además de esa brecha entre maternidad real y deseada hay una tendencia en todos los países de nuestro entorno, que es la postergación de la maternidad. Este hecho contribuye a la caída de la natalidad ya que hay muchas mujeres que cuando quieren ser madres ya no pueden por su edad. De todos los países de nuestro alrededor, España es el país de la Unión Europea en el que las mujeres son madres más tarde ${ }^{10}$.

Entre las barreras socioeconómicas que se encuentran hombres y mujeres para tener los hijos deseados en el momento deseado, están

Studies 58, no. 2 (2004), pág. 161. Se apoyan en este estudio LÓPEZ LÓPEZ, M.T. y GONZÁLEZ HINCAPIE, V. "Tener hijos, ¿forma parte del proyecto vital de los madrileños?", Maternidad, paternidad y trabajo remunerados en la Comunidad de Madrid 2017, pág. 5.

8 LÓPEZ LÓPEZ, M.T., "El reto demográfico, ¿está la solución en políticas públicas "natalistas?", Artículo publicado en ABC 10 de junio de 2017.

9 CHINCHilla, N., Maternidad y trayectoria profesional. Análisis..., cit., pág. 42.

10 Según la última estadística publicada por EUROSTAT el 1 de agosto de 2019, España es el país europeo con mayor proporción de madres primerizas de $40 \mathrm{o}$ más años. Así, el 8,8\% de las mujeres que dan a luz por primera vez tienen 40 años o más. Según cifras del Instituto Nacional de Estadística, en España han aumentado en los últimos diez años un 63\% los nacimientos de madres que han cumplido 40 años o más. 
las escasas ayudas a las familias y a la maternidad y paternidad ${ }^{11}$. Estas situaciones son reversibles, ya que sólo requieren de la voluntad política de poner en valor una cuestión que afecta a una gran mayoría social. Una de las maneras que tienen los gobiernos de demostrar fácilmente la prioridad que conceden a esta institución social es dotarla de los necesarios recursos, ampararla mediante leyes oportunas, e institucionalizar un tema que, más que nunca, se ha vuelto prioritario ${ }^{12}$.

El papel de la familia en la resolución de estos problemas sociológicos ante los que nos encontramos es fundamental, por eso los poderes públicos y las leyes deben tomar conciencia y poner en valor a la institución de la familia, ayudándola, protegiéndola e impulsando su indiscutible papel en nuestra sociedad ${ }^{13}$.

De este modo, poner en valor la institución familiar es un cometido que se puede y se debe hacer desde todos los ámbitos de la sociedad, no son únicamente los individuos, ni las empresas, ni los poderes públicos, sino todos ellos a la vez son los que deben proteger a la familia y garantizar su desarrollo. Sin embargo, es evidente, que las barreras sociopolíticas y las escasas ayudas con las que cuenta la familia son problemas que pueden solucionar los poderes públicos a través de sus leyes y ayudas a la maternidad, a la paternidad y a la familia, institucionalizando las políticas familiares y fomentando la implicación de todos los partidos políticos ${ }^{14}$.

11 "El principal incentivo a la natalidad que demandan las mujeres al Estado es el aumento de la duración del permiso de maternidad/paternidad. Por grupos de edad, los mayores porcentajes se dan entre las mujeres de 30 a 34 años $(31,1 \%)$ y de 35 a 39 años $(29,1 \%)$. El segundo incentivo a la natalidad más importante para las mujeres menores de 40 años es la flexibilidad en el horario de trabajo para padres y madres con niños pequeños. Y, para las de 40 años y más, la segunda medida más importante es una asignación para las familias con hijos a cargo menores de 18 años". Instituto Nacional de Estadística. Encuesta de Fecundidad. 28 de noviembre de 2018 , pág. 1 . 50.

12 CHINCHILla, N. Maternidad y trayectoria profesional. Análisis ...., cit., pág.

13 "Una fuerte microsolidaridad en el seno de las familias manifestada en un generoso apoyo material y afectivo entre sus miembros es responsable en gran medida de un alto nivel de satisfacción vital entre sus ciudadanos". SALIDO, O. y MORENO, L.. Política y Sociedad, 2007, Vol. 44 Núm. 2: 101-114.

14 "La conciliación de la vida laboral y familiar es un objetivo de interés general, que afecta y preocupa a todos y sobre la que se tiene que trabajar conjuntamente, siendo varios los intervinientes que participan en esta materia, pudiéndose distinguir entre otros a: la Administración Pública, las empresas, los trabajadores, las familias, el sistema educativo y la sociedad en su conjunto." COMISIÓN DE IGUALDAD, Perspectiva empresarias sobre la conciliación de la vida laboral y familiar, Versión actualizada. Octubre 2017, pág. 16.

(C) UNED. Revista de Derecho UNED, núm. 26, 2020 
A pesar de ser la familia la institución más valorada por los españoles, existe una percepción mayoritaria del escaso reconocimiento que se presta en España a las familias. Y esto tiene mucho que ver con la escasez de ayudas y una visión distorsionada del papel que desempeña ${ }^{15}$.

El Foro Español de la Familia elaboró en el año 2011 un documento con 50 Medidas de Política Familiar. En el encabezamiento del documento establece unas premisas que reflejan el valor de la institución familiar. Una de ellas asegura que "la familia debe estar respaldada en su tarea de tener hijos y de ayudarles a transformarse en ciudadanos responsables. Esto exige del Estado -de las diferentes Administraciones, debidamente coordinadas entre sí- la definición de una política pública con perspectiva de familia. Esta política con perspectiva de familia debe apoyar a la familia como un bien en sí mismo y no solo con políticas sociales de lucha contra la pobreza o de apoyo a los miembros de la familia individualmente considerados".

\section{PROPUESTAS PARA PROTEGER LA FAMILIA, LA MATERNIDAD Y LA CONCILIACIÓN}

\section{IV.A. PLANTEAMIENTO}

Como ya hemos señalado la necesidad de proteger la maternidad y la familia nos conduce a defender que se implanten políticas familiares que protejan la maternidad, impulsen la natalidad, fomenten la conciliación ${ }^{16}$ y en definitiva protejan la institución familiar. A continuación, expondremos algunas medidas de política fiscal que nos parecen que podrían contribuir a este reto centrándonos en esta ocasión en el IRPF, aunque en nuestra opinión es necesario una revisión del resto de impuestos de nuestro sistema tributario a la luz de estos valores.

15 CHINCHILlA, N., Maternidad y trayectoria profesional. Análisis ...., cit., pág. 50.

16 "La conciliación de la vida familiar y laboral se ha convertido en los últimos años en un fenómeno de gran actualidad, tanto desde el punto de vista social como político. Su centralidad radica en que la conciliación se hace necesaria para un número creciente de familias". CANÓVAS, A., ARAGÓN, J., ROCHA, F., "Las políticas de conciliación de la vida familiar y laboral en las Comunidades Autónomas". Cuadernos de Relaciones Laborales, Vol. 23, núm.1, 2005, pág. 74. 


\section{IV.B. MÍNIMO PERSONAL Y FAMILIAR}

En relación con el mínimo personal y familiar, tres son nuestras propuestas: (i) La primera es rescatar la implementación de los mínimos en la base, para así, gravar efectivamente la renta disponible del contribuyente una vez éste haya atendido a sus necesidades personales y familiares; (ii) la segunda es instrumentar un sistema de actualización de los mínimos que atienda a la inflación; (iii) y la tercera consiste en aumentar las cuantías, dado que sus importes son totalmente insignificantes (baste preguntar a cualquier padre si para mantener con dignidad y atender todas las necesidades de su hijo le bastan 2.400 euros).

En la Ley 40/1998 se estableció un mecanismo de "mínimos personales y familiares" que operaban sobre la base. La cuantía de estos mínimos minoraba la base imponible, de forma que solo la renta excedente era gravada. Así, el cálculo de la "capacidad de pago del contribuyente" se producía con carácter previo a cualquier otro sobre la progresividad del impuesto.

Esta forma de aplicar los mínimos personales y familiares fue muy cuestionada. Mediante escrito ante el Tribunal Constitucional el día 10 de marzo de 1999, ochenta y nueve diputados del Congreso, interpusieron recurso de inconstitucionalidad. Para aquellos diputados recurrentes, esta forma de aplicar los mínimos desde el punto de vista de la capacidad económica, constituía una regresión. Aseguraban que el mínimo personal y familiar que se aplica a reducir la parte general de la base imponible y no de la cuota, provoca que, siendo iguales las circunstancias personales y familiares, cuanto más elevadas son las rentas de los sujetos pasivos más aumenta la desgravación por dichas circunstancias.

Sin embargo, esta idea de está en nuestros días completamente superada. El Tribunal Constitucional se pronunció al respecto en la Sentencia 19/2012, de 15 de febrero, explicando cómo en términos porcentuales, implementar los mínimos personal y familiar en base, tiene un efecto más favorable cuanto menor es la base imponible sobre la que se aplica $\left(\mathrm{FJ} 4^{\circ}\right)$.

La Ley 35/2006 optó por cambiar el sistema. Si bien se respetaron los mínimos personales y familiares, se estableció un mecanismo en el que se desgravan mediante una detracción en la cuota del tributo equivalente a la carga que directamente generaba, pero valorada a los tipos más reducidos de la tarifa. 
La Ley 26/2014 mantuvo el sistema de mínimos personales y familiares en la cuota. El Informe de la Comisión de Expertos ${ }^{17}$ argumentó que mantenían esta opción, no porque consideraran que fuera el lugar idóneo para implementarse ni porque reconocieran aquella mal interpretada regresividad, sino "para no poner en riesgo la capacidad recaudatoria del impuesto, los mínimos personales y familiares, revisados en sus actuales cuantías, deberían seguir descontándose en la cuota conforme al procedimiento establecido en la vigente Ley del IRPF, pasando a descontarse de los ingresos netos totales cuando la consolidación presupuestaria estuviese garantizada, para respetar de ese modo el concepto de capacidad económica o capacidad de pago del contribuyente".

Desde nuestro punto de vista, la primera medida que tendríamos que tomar sería recuperar la implementación de los mínimos personal y familiar en la base. El profesor Herrera Molina señala que "es preferible que los mínimos exentos operen en la base imponible (en lugar de hacerlo en cuota) pues su función es determinar correctamente la capacidad económica gravable"18. Se podría corregir la supuesta pérdida de progresividad a través de correcciones en la tarifa pero no renunciamos a la idea clara de que los mínimos deben operar en la base si lo que se pretende es gravar efectivamente la capacidad económica real. En este sentido, nos parece relevante la reflexión que realiza Marín-Barnuevo ${ }^{19}$ : "los rendimientos utilizados para satisfacer las necesidades vitales mínimas de las personas y de su familia no son indicativos de capacidad económica, y esto con independencia de la suma total de los ingresos". Así, dos contribuyentes que tengan los mismos rendimientos netos, tendrán diferente capacidad económica en función del número de hijos que tengan o si tienen algún hijo con discapacidad. Nada tiene que ver la capacidad económica de un padre de familia numerosa, con la capacidad económica de una persona soltera, aunque los rendimientos netos de ambos sean exactamente los mismos.

Además, nos gustaría poner de manifiesto que la existencia de los mínimos personales y familiares, no debe entenderse como una

17 LAGARES CALVO, M. Informe de la Comisión de Expertos para la Reforma del Sistema Tributario Español, Ministerio de Hacienda, Madrid, 2014, págs. 147148.10.

18 HERRERA MOLINA, P.M., "Las circunstancias personales y familiares en el IRPF a la luz de la reforma tributaria”. ECJ Leading Cases, https://ecjleadingcases. wordpress.com/2014/10/13/pedro-herrera-las-circunstancias-personales-y-familiares-en-el-irpf-a-la-luz-de-la-reforma-tributaria/

19 MARÍN-BARNUEVO FABO, D., La Protección del mínimo existencial en el ámbito del IRPF, Madrid, Colex, 1996. 
ayuda o como un beneficio social, sino que es una exigencia de justicia tributaria. Para determinar la capacidad económica de un contribuyente, se deben minorar los ingresos íntegros en los mínimos personal y familiar y en los gastos deducibles. En palabras del profesor Herrera Molina "la reducción de tales mínimos de la base imponible resulta tan justificada como lo es la deducción de los gastos deducibles de los ingresos íntegros" 20 .

Según hemos señalado anteriormente, también sería deseable que se incrementaran las cuantías y que se instrumentara una forma de actualización automática de las mismas para que éstas no quedaran obsoletas con el paso del tiempo, o que se determinaran anualmente en los Presupuestos Generales del Estado, con un incremento, como mínimo, igual al incremento del IPC.

\section{IV.C. PRESTACIÓN UNIVERSAL POR NACIMENTO}

Nuestra propuesta consiste en recuperar la deducción por nacimiento o adopción en el IRPF y la prestación económica de pago único de la Seguridad Social por nacimiento o adopción que fue aprobada en la Ley 35/2007 de 15 de Noviembre.

El conocido "cheque bebé" lo percibieron en España todas aquellas personas que tuvieron un hijo entre el 1 de Julio de 2007 y el 31 de Diciembre de 2010. Fue una medida que muchos tacharon de electoralista, sin embargo, se configuró como una ayuda directa a las familias, a la conciliación, al impulso de la natalidad y tuvo una muy buena percepción por parte de los padres, ya que todos, pudieron percibirla, no sólo aquellos que son contribuyentes del IRPF, porque se regula con una doble naturaleza: (i) como un beneficio fiscal que minora la cuota diferencial del IRPF y se puede pedir de forma anticipada, y (ii) como prestación de la Seguridad Social en su modalidad no contributiva. Este hecho es relevante y nos parece interesante, y es que por ejemplo, los mínimos personal y familiar que comentamos en el epígrafe anterior, no pueden ser aprovechados por todas las personas; aquellas que no son contribuyentes o los que no tienen rentas suficientes para absorberlos, los pierden. Sin embargo, este cheque bebé sí se configuró como una ayuda, como un beneficio fiscal para todos españoles que tuvieron un hijo durante aquellos ejercicios en los que estuvo vigente.

20 HERRERA MOLINA, P.M. La urgente reforma de los mínimos vitales (Mejoras Técnicas del IRPF, V). ECJ Leading Cases, https://ecjleadingcases.wordpress. com/2018/12/30/la-urgente-reforma-de-los-minimos-vitales-mejoras-tecnicas-irpf-v/

(C) UNED. Revista de Derecho UNED, núm. 26, 2020 
Cuando la fiscalidad establece estos mecanismos que ayudan de forma directa y universal a las personas que están formando una familia, teniendo hijos y educándolos para que sean el futuro de nuestra sociedad, se pone de manifiesto que nuestro sistema tributario verdaderamente tiene otros fines que no son sólo los recaudatorios, pues protege valores consagrados en la Constitución y que la finalidad extrafiscal de los tributos también es relevante (en este caso, la protección de la natalidad).

Por todo lo anterior, proponemos que se instaure de nuevo el cheque bebé con la misma doble naturaleza y la misma forma de instrumentación que el regulado en la Ley 35/2007 de 15 de noviembre ${ }^{21}$.

Es preciso traer a colación aquí que algunos ayuntamientos y otras entidades locales conceden "ayudas por nacimiento o adopción”. Así, por ejemplo el Ayuntamiento de Pozuelo de Alarcón que concede ayudas por nacimiento o adopción con el objetivo de "fomentar la natalidad", estas ayudas tienen el carácter de subvención. Es deseable que se tome conciencia de la gran ayuda que supone para las familias y el buen impacto social que tienen estas subvenciones.

\section{IV.D. EXENCIÓN DE LA PRESTACIÓN POR MATERNIDAD}

La Sentencia 1462/2018 de 3 de Octubre, el Tribunal Supremo declaró exenta la prestación por maternidad de la Seguridad Social,

21 Según la Exposición de Motivos de la Ley 35/2007:"Esta nueva prestación tiene una doble naturaleza. Para las personas que realicen una actividad por cuenta propia o ajena por la cual estén dadas de alta en la Seguridad Social en el momento del nacimiento o la adopción, o hubieran percibido en el período impositivo anterior rendimientos o ganancias de patrimonio, sujetos a retención o ingreso a cuenta, o rendimientos de actividades económicas por los que se hubieran efectuado los correspondientes pagos fraccionados, la prestación adquiere el carácter de beneficio fiscal y minora la cuota diferencial del Impuesto sobre la Renta de las Personas Físicas, pudiendo percibirse de forma anticipada. Por el contrario, de forma subsidiaria, para las personas que no tienen derecho al beneficio fiscal antes indicado por no encontrarse en la situación descrita, el pago adquiere la naturaleza de prestación de Seguridad Social, en su modalidad no contributiva"

El artículo 3 Ley 35/2007 establece las cuantías: a) Una deducción de 2.500 euros anuales en el Impuesto sobre la Renta de las Personas Físicas aprobado por la Ley 35/2006, de 28 de noviembre, del Impuesto sobre la Renta de las Personas Físicas y de modificación parcial de las leyes de los Impuestos sobre Sociedades, sobre la Renta de no Residentes y sobre el Patrimonio (...).

Esta deducción podrá percibirse de forma anticipada y se aplicará con cargo al tramo estatal del impuesto.

b) Una prestación no contributiva de la Seguridad Social de 2.500 euros, en el supuesto de no cumplir los requisitos establecidos en la letra a) anterior. 
y aunque no se pronunció sobre la prestación percibida por paternidad, la Agencia Tributaria automáticamente asumió su similitud, y en el propio formulario para solicitar la devolución que instrumentó en la página web de la Agencia Tributaria, incluyó la paternidad sin la necesidad de ningún otro pronunciamiento.

Según hemos visto en el epígrafe de la introducción de este artículo, dicha prestación de paternidad percibida de la Seguridad Social y otras prestaciones análogas de paternidad y maternidad que son percibidas por los profesionales independientes de sus mutualidades o por funcionarios públicos, también están exentas en virtud de la nueva redacción del artículo 7 h) LIRPF dada por el Real Decreto - Ley 27/2018.

Desde nuestro punto de vista, la exención de la prestación por maternidad percibida de la Seguridad Social es una medida regresiva, que beneficia a las rentas más altas. La prestación por maternidad que otorga la Seguridad Social se concede según el principio de cotización, es decir que cuanto más altas son las rentas de los contribuyentes, más cotizan y mayor es su prestación por maternidad que la mencionada Sentencia 1462/2018 declara exenta. Esto supone un ahorro fiscal mucho más cuantioso e importante para los perceptores de rentas altas. Esta medida regresiva va contra el principio de igualdad tributaria que rige o debe regir todo el ordenamiento tributario español.

De todos modos, valoramos el esfuerzo que está haciendo el legislador para, en palabras del Tribunal Supremo, "reconocer la maternidad como una situación protegida" (así como la adopción, el acogimiento familiar y la familia en sentido amplio) y nos parece muy oportuno y deseable que se implanten políticas familiares que protejan la maternidad, impulsen natalidad, fomenten la conciliación y en definitiva protejan la institución familiar.

Nuestra primera propuesta sería, para que técnicamente sea correcta esta exención por maternidad, implantar algún mecanismo que neutralice este efecto regresivo, por ejemplo, establecer un límite máximo exento, y que el exceso sí quede sometido a tributación.

Nuestra segunda propuesta en este ámbito, es la siguiente: si está exenta la prestación por maternidad, por coherencia tributaria, también deberían estar exentos los otros supuestos relacionados con la maternidad tales como la prestación percibida por riesgo para el embarazo y la prestación percibida durante la lactancia natural (que 
están contemplados en la LSS en el mismo artículo que la prestación por maternidad).

\section{IV.E. PROPUESTA DE DEDUCCIONES FAMILIARES (ESTATALES Y UNIVERSALES)}

Las deducciones de la cuota son un instrumento frecuentemente utilizado para incentivar conductas coherentes con los objetivos de política económica o social sin distorsionar la progresividad. Por eso nos parece que es un mecanismo idóneo para utilizarlo en la protección a la familia. Las deducciones que proponemos deberían ser universales $^{22}$, es decir, para todos los contribuyentes, con independencia de su nivel de renta y estatales, ya que es el Estado el que debe apoyar a la familia, y no únicamente una Comunidad Autónoma o una entidad local concreta. Son deducciones, que pretenden proteger la conciliación de la vida personal y profesional, y en definitiva, a la familia.

Proponemos las siguientes deducciones:

IV. E. 1. Deducción por reducción de jornada para conciliar vida personal y vida profesional

Muchas personas cuando sus hijos son pequeños o tienen alguna necesidad especial, desearían pedir una jornada reducida en su trabajo para poder tener más tiempo para atenderles. Sin embargo, la pérdida económica que tal reducción de jornada lleva aparejada, no se lo permite. Sería deseable, que todas aquellas personas que quisieran disfrutar de una jornada reducida pudieran acceder a la misma y podría ser interesante que, vía deducciones, esa pérdida económica no fuera tan determinante.

22 "Una de les características más marcadas de la política familiar en la Unión Europea es su universalismo. Es decir, va dirigida a la inmensa mayoría de familias con hijos menores a cargo. Así, la tendencia dominante en los países europeos es que los subsidios familiares sean de carácter universal y se paguen a todas las familias, independientemente de su nivel de renta. Pero en los últimos tiempos, debido a las restricciones presupuestarias, también se han ido afirmando otras orientaciones en el sentido de otorgar un número creciente de prestaciones bajo condición de recursos". FLAQUER, L.. Políticas familiares en una perspectiva comparada. Colección Estudios Sociales. Núm. 3. Fundación La Caixa, pág. 151. 
En este estudio estamos centrándonos el IRPF, pero es evidente que en otros impuestos como por ejemplo el Impuesto sobre Sociedades, también sería conveniente establecer deducciones correlativas para incentivar desde todas las perspectivas este comportamiento que facilita la conciliación, estableciendo deducciones para empresas familiarmente responsables.

Somos conscientes de que esta propuesta no solucionaría algunos otros factores que imposibilitan a muchos trabajadores acceder a una jornada reducida en pro de su familia, ya que todavía hoy en muchas empresas, tal reducción está mal vista desde el punto de vista del compromiso con el trabajo, y por desgracia a muchas personas se le acaba la carrera profesional cuando piden una reducción de jornada. Por ello, no sólo es el Estado, a través de sus leyes, sino también las familias y todos los trabajadores debemos ser agentes de cambio y promover un ambiente laboral en el que lo importante sean los objetivos y el trabajo bien hecho, y que el trabajo en equipo facilite esas jornadas para aquellos que lo soliciten.

IV. E. 2. Deducción por gastos de guardería. Deducción por gastos de ayuda doméstica

Esta deducción de gastos por guardería existe con carácter autonómico en algunas Comunidades, como por ejemplo en Aragón. Sin embargo, la deducción tiene un límite de 250 euros y solo la pueden aplicar algunos contribuyentes (aquellos cuya suma de la base liquidable general y de la base liquidable del ahorro sea inferior a 35.000 euros en declaraciones individuales e inferior a 50.000 euros en declaraciones conjuntas, siempre que la base imponible del ahorro, sea cual sea la modalidad de la declaración, no supere 4.000 euros). Estas deducciones existentes son claramente insuficientes. Cualquier persona que tenga un hijo en una guardería sabe que con 250 euros no paga ni la primera mensualidad.

Además, recientemente, la ley 6/2018 de Presupuestos Generales del Estado y el Proyecto de modificación del Reglamento del IRPF, ha instaurado un cheque guardería que mejora la deducción por maternidad; el importe de la deducción son 83,33 euros por mes (con un máximo de 1.000 euros) y no es posible solicitar su percepción de modo anticipado.

Desde nuestro punto de vista, la introducción del cheque-guardería en la deducción por maternidad no es técnicamente correcta. Nada tiene que ver una con otra, ni siquiera la forma de percepción 
y no contribuye en absoluto con el ideal de simplicidad de nuestro sistema tributario. Sin embargo, a pesar de todo ello, nos parece acertado que el legislador sea consciente de que ayudar a las familias con el servicio de guardería de sus hijos menores de tres años. Es un primer paso para proteger la maternidad, la paternidad, la conciliación y la familia. Nuestra propuesta sería que existiera una deducción por gastos de guardería estatal, independiente de la deducción por maternidad, para todas las familias con hijos menos de tres años, y otra deducción exacta en cuantía y en naturaleza, para aquellas familias que en lugar de llevar a sus hijos a un centro especializado, prefieren dejarles en esas edades tempranas en casa a cargo de sus empleadas del hogar.

IV. E. 3. Deducción por gastos de ayuda doméstica

En la Comunidad de Madrid existe una deducción por cuidado de hijos menores de tres años. La cuantía es el 20 por 100 de las cuotas ingresadas por el contribuyente por cotizaciones al Sistema Especial de Empleados de Hogar del Régimen General de la Seguridad Social, con el límite de deducción de 400 euros anuales (en el caso de familias numerosas la cuantía asciende al 30 por ciento de las cuotas ingresadas y el límite a 500 euros anuales). Esta deducción no es universal; tiene un límite que se calcula en función del número de miembros que integran la unidad familiar, este mecanismo, que utiliza la comunidad de Madrid en otras deducciones como la deducción por gastos educativos que veremos más adelante, nos parece muy interesante y se ajusta más desde nuestro punto de vista al principio de igualdad, ya que tiene en cuenta la composición real de las familias.

Además, y desde un punto de vista extrafiscal, ayuda a incentivar la contratación de personal para el hogar que esté dado de alta en la Seguridad Social.

Proponemos que esta deducción se amplíe al ámbito estatal, y que, de tener un límite, el mismo tenga en cuenta el número de miembros de la unidad familiar, tal y como lo instrumenta la Comunidad de Madrid. 
IV. E. 4. Deducción por gastos de escolaridad

En la Comunidad de Madrid existe una deducción autonómica por gastos educativos; la misma engloba gastos de escolaridad, gastos de uniforme y gastos de enseñanza de idiomas. Esta deducción tiene algo que nos parece muy interesante, que hemos comentado también en el epígrafe anterior, y es que no siendo universal (no todos tienen derecho a cobrarla), sin embargo establece un límite que tiene en cuenta el número de miembros de la unidad familiar ("Sólo tendrán derecho a aplicar la deducción los contribuyentes cuya base imponible (suma de la base imponible general y del ahorro) junto a la del resto de los miembros de la unidad familiar, no supere el importe resultante de multiplicar 30.000 euros por el número de miembros de la unidad familiar").

Esta deducción apoya a la familia, y pretende de algún modo, colaborar con el gasto que hacen los padres para la educación de sus hijos. Es una deducción que nos parece interesante y que proponemos se amplíe al ámbito estatal. La educación y la familia son dos pilares de nuestra sociedad que miran al futuro; cuidarlos, protegerlos y potenciarlos, tienen que ser una prioridad para el legislador.

\section{E. 5. Deducción por transporte escolar}

No tenemos conocimiento de que exista esta deducción en alguna comunidad autónoma, pero en nuestra opinión sería de gran interés. Su objetivo sería facilitar la conciliación. Muchos padres llevan a sus hijos al colegio o a la guardería porque el transporte escolar es muy caro, esos padres o bien llegan más tarde a trabajar, o dejan a sus hijos en el colegio mucho tiempo antes de que empiece el horario escolar (de hecho últimamente existe un "horario flexible" para los niños que permite que les dejen una hora antes, o que les recojan una hora después de empezar el horario habitual, por supuesto, como norma general, con un cargo económico adicional). Si el transporte escolar no tuviera los costes tan elevados como tiene, muchos padres, utilizarían la ruta para llevar a sus hijos al colegio, lo cual sería bueno para todos, familias y empresarios. Los niños no entrarían tan temprano y los padres llegarían antes a sus puestos de trabajo, también podrían salir antes.

Proponemos incluir este concepto en la deducción por gastos educativos y nos parece que sería una buena medida para promover la conciliación 


\section{F. DEDUCCIONES DE LA CUOTA DIFERENCIAL}

Las deducciones de la cuota diferencial que prevé nuestro ordenamiento jurídico, se han erigido como uno de los instrumentos a través de los cuales el legislador fiscal ha dado un paso al frente a la hora de proteger a las familias. Para muchos expertos en la materia estas deducciones no son técnicamente correctas y perjudican la simplicidad del impuesto ${ }^{23}$. Sin embargo, es de nuevo un instrumento que cuenta con una buena acogida por parte de los contribuyentes y la posibilidad de solicitar su abono anticipado hace que los perceptores de la misma sean conscientes de que se trata de una medida que apoya a las madres trabajadoras con hijos menores de tres años, a las familias numerosas y a las familias con hijos discapacitados a su cargo. Estas deducciones a la cuota diferencial, ayudan al contribuyente a percibir esa finalidad extrafiscal de los tributos a través de lo que se configura como una ayuda directa a las familias.

Estas deducciones, conocidas como "impuestos negativos", son básicamente tres: la deducción por maternidad, la deducción para familias numerosas y la deducción por hijo discapacitado a cargo. A continuación haremos algunos apuntes para sugerir algunos modos de perfeccionarlas o ampliarlas.

\section{F. 1. Deducción por maternidad}

Esta deducción la perciben las madres con hijos menores de tres años que realicen una actividad por cuenta ajena o propia y que están dadas de altas en el Régimen de la Seguridad Social o en una Mutualidad alternativa. La deducción asciende a 1.200 euros anua-

23 Sobre la valoración de esta figura de los impuestos negativos, hay varias opiniones ya que algunos expertos aseguran que implementarlos supone alterar la naturaleza propia del Impuesto. Se discute si es más eficaz y respetuoso con la naturaleza de los impuestos acometer este tipo de ayudas a través de políticas de gasto. El profesor Herrera Molina considera criticable el condicionamiento de estas deducciones a las cotizaciones sociales y en la línea de los defensores de acometer estas medidas de política familiar mediante otros instrumentos asegura que "sería preferible que la situación de las familias numerosas se tuviera en cuenta mediante la financiación de servicios y subvenciones". Así, asegura que "convendría ir sustituyendo una parte de la cuantía de los mínimos mediante el establecimiento de servicios (...) como por ejemplo un servicio gratuito de guarderías de 0 a 3 años". Cfr. HERRERA MOLINA, P.M., "Las circunstancias personales y familiares en el IRPF a la luz de la reforma tributaria". ECJ Leading Cases, https://ecjleadingcases.wordpress.com/2014/10/13/pedro-herrera-las-circunstancias-personales-y-familiares-enel-irpf-a-la-luz-de-la-reforma-tributaria/ 
les con la posibilidad de solicitar su abono anticipado a razón de 100 euros mensuales.

Esta deducción, como ya se ha dicho, desde el 1 de enero de 2018 se ha visto incrementada en 1.000 euros anuales cuando se hayan satisfecho gastos de custodia del hijo menor de tres años en guarderías o centros especializados. En nuestra opinión, no es técnicamente correcto insertar como un incremento de la deducción por maternidad, esta deducción por gastos de guardería. En este sentido, creemos que debería más bien configurarse como una deducción separada. Pero nos gustaría añadir aquí que, mientras se instrumenta una deducción individual y particular sobre gastos de guardería y de custodia de hijos menores de tres años, podría tal incremento de la deducción por maternidad por gastos de guardaría tener en cuenta no solo los gastos satisfechos en guarderías y centros especializados, si no también, gastos de custodia por personal del hogar.

Además de esto último, nuestras propuestas en relación con esta deducción por maternidad son las siguientes:

a. Ampliar la deducción a las madres no trabajadoras, o madres que no tienen un trabajo profesional fuera de su casa. Sería una forma de reconocer el trabajo que esas madres realizan por su familia y en definitiva por la formación de las mujeres y de los hombres del futuro ${ }^{24}$. Esto tiene una posible crítica y es que esta deducción lo que pretendía originariamente era proteger la conciliación, por eso se instrumentaba para que la percibieran las madres trabajadoras, pero sugerimos un cambio de enfoque, y podría ser una medida de protección a la familia.

b. Ampliar en duración y que no solo la percibieran las madres de hijos menores de de 3 años sino por ejemplo, que las percibie-

24 "El trabajo doméstico, tan desprestigiado en ocasiones a favor del trabajo realizado en el mercado laboral, cumple un papel esencial: tiene un valor invisible pero real, supone un ahorro para los servicios sociales públicos, y, por su misma naturaleza, desarrolla en la persona habilidades y competencias relacionadas con el servicio y la convivencia. Por este motivo, también el varón se ve beneficiado personalmente cuando participa en las tareas del hogar, lo que repercute en la familia, en los hijos, en las empresas y en el conjunto de la sociedad.

De acuerdo con el estudio realizado por el Instituto Català de les Dones (ICD) y el Observatorio Mujer, Empresa y Economía (ODEE) de la Cámara de Comercio de Barcelona en febrero de 2017, si se pagase a precio de mercado, el valor del trabajo doméstico no remunerado incrementaría el Producto Interior Bruto (PIB)1 de Cataluña, como mínimo, un 23,4\%. Dicho estudio pone de manifiesto que las mujeres son las que realizan una mayor aportación a la sociedad, a través de la economía doméstica y del cuidado (67\% frente al 33\% de los hombres)." CHINCHILLA, N. Maternidad y trayectoria profesional. Análisis ...., cit., pág. 12. 
ran todas las madres hasta que sus hijos cumplieran 12 años (esta es la edad que fija el Estatuto de los Trabajadores cómo límite para que una trabajadora pueda solicitar la reducción de jornada para el cuidado de sus hijos).

c. Ampliar en cuantía. Actualmente se concede 100 euros por hijo menor de tres años, esta cantidad es acumulativa, si se tienen dos hijos menores de tres años, se perciben 200 euros. Nosotros no sugerimos únicamente que se amplíe la cuantía a menores de 12 años, sino que además, la cuantía aumente por hijo, es decir, 100 euros por el primer hijo menor de 12 años y luego 50 euros más por cada hijo menor de esta edad establecida.

d. Ampliar esta deducción a la paternidad. Con esta medida duplicaríamos la ayuda a las familias, e iríamos en la línea de la corresponsabilidad ayudando no solo a las madres, sino también a los padres.

IV. F. 2. Deducción para familias numerosas o personas con discapacidad a cargo

Esta deducción funciona de forma análoga a la deducción por maternidad arriba indicada; minora la cuota diferencial con independencia de que esta sea positiva o negativa y se puede pedir el abono anticipado.

Según establece la ley está prevista para contribuyentes con mayores cargas familiares, y comprende cinco deducciones:

- Deducción por cada descendiente con discapacidad.

- Deducción por cada ascendiente con discapacidad.

- Deducción por cónyuge no separado legalmente con discapacidad.

- Deducción por familia numerosa.

- Deducción por ascendiente, separado legalmente o sin vínculo matrimonial, con dos hijos sin derecho a percibir anualidades por alimentos.

La cuantía es de 1.200 euros anuales para todas las deducciones relacionadas con la discapacidad. La cuantía de la deducción por familia numerosa de categoría general, es también de 1.200 euros anuales, y de 2.400 euros para familias numerosas de categoría es- 
pecial. Recientemente, se ha aprobado que además, estas deducciones de las familias numerosas se pueden incrementar en 600 euros anuales por cada uno de los hijos que formen parte de la familia numerosa que exceda del número mínimo de hijos exigido para que esa familia tenga la condiciones de familia numerosa general o especial (es decir, una familia de cuatro hijos, que según la Ley de Protección a las Familias Numerosas es una familia numerosa de categoría general, podrá aplicarse una deducción de 1.200 euros más 600 euros, del mimos modo que una familia con seis hijos, podrá aplicarse una deducción por 2.400 euros más 600 euros).

Estamos ante unas deducciones que, según hemos señalado anteriormente, tienen muy buena acogida entre estas familias con necesidades especiales: Nos parece que deben seguir en vigor y sería deseable que se fueran incrementado con el coste de la vida, igual que señalamos en el epígrafe de los mínimos personal y familiar con el objeto de que no queden obsoletos por el paso del tiempo.

Otra propuesta es mantener la deducción incrementada de 2.400 euros para aquellas familias que siendo numerosas de categoría especial pierden la condición de especial, cuando sus hijos mayores cumplen 25 años. Proponíamos mantener la deducción incrementada hasta que los hijos pequeños cumplieran la edad requerida. Esto es ya aplicable gracias a la reciente Sentencia del Tribunal Supremo 951/2019 de 25 de marzo, que establece como doctrina legal que "debe interpretarse (el artículo 6 de la Ley de Familias Numerosas) en el sentido de que el Título de Familia Numerosa en la circunstancia a que se refiere ese párrafo sigue en vigor no sólo en su existencia sino, además, en la categoría que antes ostentara" es decir, que por el hecho de que los hijos mayores cumplan 25 años y ya no se entiendan dentro de la familia numerosa, la condición y el titulo de familia numerosa de categoría especial debe seguir vigente mientras al menos uno de los hijos cumpla con las condiciones establecidas en la Ley de Familias numerosas, a saber, edad (21 o 25 años), dependencia y convivencia.

\section{NECESIDAD DE ANÁLISIS CUANTITATIVOS SOBRE LOS EFECTOS ECONÓMICOS DE LAS MEDIDAS PROPUESTAS}

Todas estas medidas que se han propuesto y analizado tienen por objeto proteger la maternidad, la paternidad, la familia ${ }^{25}$ y la con-

25 "Tener una familia no es una carga a compensar, sino una ventaja para la comunidad, lo que la hace acreedora de apoyo". FLAQUER, L.. Políticas familiares en

(C) UNED. Revista de Derecho UNED, núm. 26, 2020 
ciliación. Ahora bien, nos parece necesario evaluar su efecto económico. Sería deseable que antes de implementarlas se hiciera un estudio cuantitativo y económico, para ver su impacto real y para analizar si cumplen con sus objetivos, a saber:

- Proteger a la institución familiar.

- Ayudar a las familias con necesidades especiales (con personas discapacitadas, monoparentales o numerosas).

- Fomentar la natalidad.

- Fomentar la conciliación.

Evidentemente no se pueden implementar todas las medidas propuestas, por eso, este estudio económico nos facilitaría la toma de decisiones sobre cuales medidas son más efectivas y cuales se podrían implementar para que verdaderamente, las medidas tomadas, consigan los objetivos para los que se diseñaron y se implementen instrumentos de política económica, fiscal y social realmente eficaces y efectivos ${ }^{26}$.

\section{CONCLUSIONES}

Las principales conclusiones a las que llegamos después de la preparación de este artículo son las siguientes:

- España atraviesa un momento delicado demográficamente hablando y todos, los ciudadanos y el legislador, deben colaborar para tomar medidas en pro de la natalidad que faciliten a las familias la posibilidad de tener los hijos que deseen y de educarlos para que sean el futuro de nuestra sociedad y de nuestro país.

- Actualmente existen muchas barreras socioeconómicas que frenan a las familias españolas a tener más hijos. Existe una

\footnotetext{
una perspectiva comparada. Colección Estudios Sociales. Núm. 3. Fundación La Caixa, pág. 46.

${ }^{26}$ Ya existen algunos estudios que miden el impacto de determinados beneficios fiscales, como por ejemplo, el impacto de la deducción por maternidad. RECIO ALCAIDE, A. y PEREZ LÓPEZ, C. "Impacto de la deducción por maternidad en la incorporación de la mujer al mercado de trabajo". Encuentro de Economía Pública 2018.

En el citado trabajo se llega a algunas conclusiones tales como que "Se deduce por tanto que un aumento en el importe del incentivo fiscal de esta medida incrementa la tasa bruta de natalidad", pág. 8.
} 
brecha entre maternidad deseada y maternidad real que carece de sentido en un país en el que la pirámide poblacional está invertida. Urge tomar medidas que no sólo darán estabilidad al país, sostenibilidad a las pensiones, sino que harán individuos más equilibrados y más felices, capaces de conciliar su deseo de ser buenos profesionales y contribuir a la economía del país con su deseo de tener hijos y de formar familias.

- Las políticas fiscales y los tributos pueden contribuir positivamente a estos objetivos de fomento de la natalidad, la conciliación, la protección a la institución familiar. Concretamente, el IRPF, impuesto personal y directo por excelencia, es susceptible de reformas que lo pueden configurar como un verdadero instrumento de protección familiar, por ejemplo a través de:

- La correcta implementación del mínimo personal y familiar en la base.

- La creación de una prestación universal por nacimiento.

- La creación de deducciones en pro de la conciliación, como deducciones por guardería, por ayuda doméstica, por gastos educativos, por transporte escolar, por reducción de jornada para el cuidado de hijos.

- El reforzamiento de las deducciones por maternidad y por paternidad, ampliando las cuantías o la duración de la deducción por maternidad, y creando una deducción por paternidad.

- Todas estas propuestas de reforma deben evaluarse y cuantificarse para implementar sólo aquellas que verdaderamente cumplan con su cometido y consigan que la natalidad aumente, la conciliación sea efectiva, la maternidad y la paternidad valores protegidos y las familias experimenten la protección, el apoyo y el reconocimiento que necesitan y merecen. 
